\title{
Mitochondrial Respiratory Chain Enzymatic Activities on Skin Fibroblasts in Patients With Mutant Glucocerebrosidase and PARK2 Genes
}

\author{
Kawinthra Khwanraj ${ }^{\mathrm{a}}$, Lulin Choubtum ${ }^{\mathrm{b}, \mathrm{c}}$, Aruchalean Taweewongsounton ${ }^{\mathrm{b}, \mathrm{c}}$, Somsak Tanrattanakorn ${ }^{\mathrm{d}}$, \\ Teeratorn Pulkes ${ }^{\mathrm{b}}$, Permphan Dharmasaroja ${ }^{\mathrm{a}, \mathrm{e}}$
}

\begin{abstract}
Background: Impairments of mitochondrial respiratory chain have been observed in skin fibroblast of patients with sporadic Parkinson's disease (PD) and PARK2 mutations. This study assesses enzymatic activity of mitochondrial respiratory chain in skin fibroblasts of Thai PD patients with glucocerebrosidase (GBA) and PARK2 mutations.
\end{abstract}

Methods: Skin fibroblasts were obtained from 14 individuals including four mutant GBA alleles, three mutant PARK2 alleles, and seven PD patients without mutations. Five age-matched healthy controls were recruited. Activities of complex I - V were assessed using enzyme-linked immunosorbent assay, and normalized with citrate synthase activity. All measurements were performed in triplicate.

Results: Complex V activity seemed to be lower in PD patients than controls. Among patients, activities of complexes I, II, IV and V seemed to be lower in the mutant group than those without mutation. Among patients with mutations, the mutant GBA group showed slightly lower activities of complexes II, IV, and V than the mutant PARK2 group.

Conclusions: A skin fibroblast culture may be a useful tool to investigate how GBA mutation leads to impairment of the energetic metabolism in patients with PD.

Keywords: Respiratory chain; Mitochondria; Fibroblast; Parkinson's disease; Parkin; GBA

Manuscript accepted for publication February 25, 2016

aDepartment of Anatomy, Faculty of Science, Mahidol University, Bangkok 10400, Thailand

bDivision of Neurology, Department of Medicine, Faculty of Medicine, Ramathibodi Hospital, Mahidol University, Bangkok 10400, Thailand

'Research Center, Faculty of Medicine, Ramathibodi Hospital, Mahidol University, Bangkok 10400, Thailand

${ }^{\mathrm{d}}$ Division of Dermatology, Department of Medicine, Faculty of Medicine, Ramathibodi Hospital, Mahidol University, Bangkok 10400, Thailand

${ }^{\mathrm{e} C}$ Corresponding Author: Permphan Dharmasaroja, Department of Anatomy, Faculty of Science, Mahidol University, Rama VI Road, Ratchathewi, Bangkok 10400, Thailand. Email: permphan.dha@mahidol.ac.th

doi: http://dx.doi.org/10.14740/jnr375w

\section{Introduction}

Parkinson's disease (PD) is the second most common neurodegenerative disease whose characteristic clinical signs include muscle rigidity, slowness of movement, rest tremors, and postural instability. While most PD cases are sporadic, mutations of several genes have been identified as a causative factor for rare familial PD. PARK2 (Parkin) mutations are known to be the most common cause of familial or early-onset PD [1]. Compound heterozygous abnormalities in the PARK2 gene have been found in skin fibroblasts isolated from patients with middle ageonset familial parkinsonism [2]. Skin fibroblasts from patients with PARK2 mutations have also demonstrated a distinct and unique mRNA expression pattern of key genes in neurodegeneration, which are different from those found in idiopathic PD and other mutations [3]. The association between PD and mutations in the acid $\beta$-glucocerebrosidase (GBA) gene has been reported [4-9]. Mechanisms, by which loss-of-function mutations of GBA lead to clinical phenotypes similar to sporadic PD, still remain elusive. The possible mechanism is that the GBA mutation compromises lysosomal protein degradation and leads to accumulation of $\alpha$-synuclein, which results in neurotoxicity [10, 11]. The reduction in the GBA activity has been observed in skin fibroblasts of PD patient with GBA mutation [12].

Functional and morphological impairments of mitochondrial respiratory chain have been observed in skin fibroblast of sporadic PD patients and PD patients with PARK2 mutations, such as lower mitochondrial complex I and $\mathrm{V}$ activities and adenosine triphosphate (ATP) production [13-15]. GBA inhibition can cause decreased adenosine diphosphate (ADP) phosphorylation, reduced mitochondrial membrane potential and increased free radical formation in the SH-SH5Y human neuroblastomal cell line [16]. Here, we investigate the enzymatic activity of mitochondrial respiratory chain in skin fibroblast cultures of Thai PD patients with PARK2 and GBA mutations, in comparison to those of controls and non-mutant PD patients.

\section{Materials and Methods}

\section{Ethics statement}

The study was approved by the Ethical Clearance Committee 


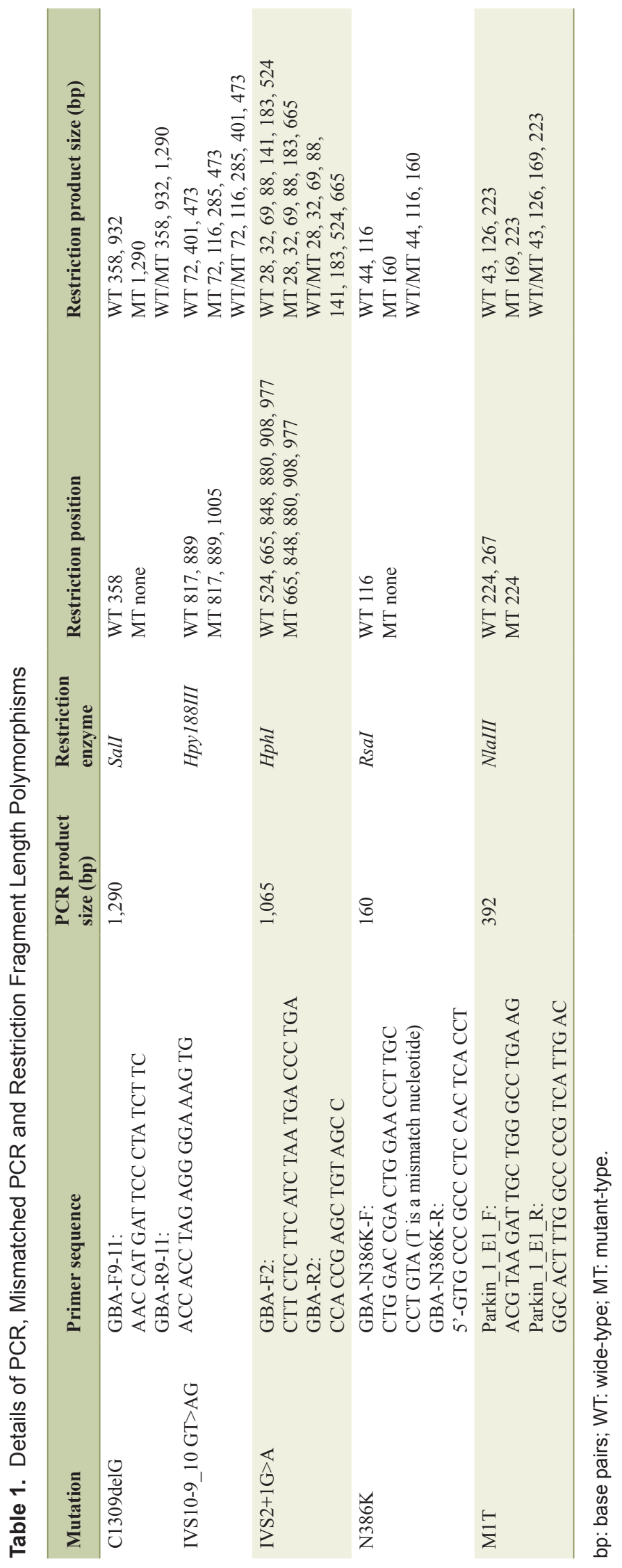

on Human Rights Related to Research Involving Human Subjects, Faculty of Medicine, Ramathibodi Hospital, Mahidol University (ID 03-54-22). All participants provided both verbal and written informed consent prior to the enrollments.

\section{Patients and controls}

The authors had recent works on a comprehensive genetic analysis of GBA [17] and PARK2 genes (unpublished data), and screening for p.R1628P, p.G2019S and p.G2385R variants of the leucine-rich repeat kinase 2 gene (LRRK2) in a large Thai PD cohort [18]. Here, we recruited the PD patients from the previous cohort for further studying on a mitochondrial respiratory chain function associated with a heterozygous allele of GBA and PARK2 mutations. Fourteen patients diagnosed with PD and five healthy age-matched controls were enrolled in this study. The patient and control groups were evaluated in the Neurology Department of the same University Hospital. All PD patients had onset of disease before the age of 50 years, and all fulfilled the UK Parkinson's Disease Brain Bank criteria [19]. Four patients carried heterozygous GBA point mutations, two had heterozygous deletion of exon 8 of PARK2, one had a heterozygous PARK2 point mutation, and seven remaining patients had no mutations in the genes studied (Table 1). Details of the identification of GBA variants have been described in our previous publication, which can distinguish functional GBA from its pseudogene [17]. To our knowledge, all but $\mathrm{p}$. IVS2+1g $>$ A mutations of the GBA were identified in only Thai PD patients [17]. The PARK2 point mutation, p.M1T, is a novel mutation identified in $0.8 \%$ of the Thai PD cohort (4/513), and absent in over 400 control subjects. In contrast, a heterozygous deletion of exon 8 of PARK2 was detected in both PD patients $(1.2 \%)$ and control group $(2 \%)$, suggesting that it may not play a pathogenic role on risk of developing PD. Controls were recruited from patients presenting with other medical conditions and had no signs of parkinsonism and were analyzed for the absence of GBA and PARK2 mutations.

\section{Skin fibroblast cultures}

Skins were obtained from the dorsal region of the inner upper arm of each PD patient or control. The biopsy specimens were maintained in tubes with Dulbecco's modified Eagle's medium (DMEM) (Gibco, Gaithersburg, MD, USA), stored at $4{ }^{\circ} \mathrm{C}$ and processed within $4 \mathrm{~h}$ in laminar flow. Fibroblast harvesting was done by explant, isolating the dermis from the epidermis with scalpels and scissors. Then dermis specimens were fragmented in small pieces. These fragments were laid onto the surface of Petri dishes containing high glucose-DMEM, penicillin (100 $\mathrm{UI} / \mathrm{mL})$, streptomycin $(100 \mathrm{mg} / \mathrm{dL})$, L-glutamine $(4 \mathrm{mM})$ and supplemented with heat-inactivated fetal calf serum. The cultures were maintained at $37^{\circ} \mathrm{C}$ in a humidified atmosphere of 95\% air and $5 \% \mathrm{CO}_{2}$. Fibroblasts were grown to confluence, harvested by trypsinization at $37^{\circ} \mathrm{C}$, washed with culture medium, resuspended with phosphate buffer, and then sonicated to obtain the cell homogenate. In all assays, fibroblast passage 
Table 2. Phenotypic and Genotypic Data of Parkinson's Disease Patients and Controls

\begin{tabular}{|c|c|c|c|c|c|}
\hline & Code & Gender & Age (years) & Causative gene & Mutation \\
\hline \multirow[t]{14}{*}{ Patients } & 1 & Male & 51 & None & - \\
\hline & 2 & Male & 57 & None & - \\
\hline & 3 & Male & 47 & None & - \\
\hline & 4 & Male & 37 & None & - \\
\hline & 5 & Male & 45 & None & - \\
\hline & 6 & Male & 46 & None & - \\
\hline & 7 & Female & 53 & None & - \\
\hline & 8 & Male & 56 & PARK2 & exon 8 deletion \\
\hline & 9 & Female & 43 & PARK2 & p.Met1Thr \\
\hline & 10 & Female & 41 & PARK2 & Exon 8 deletion \\
\hline & 11 & Male & 56 & $G B A$ & IVS11-9,10 gt>ag \\
\hline & 12 & Female & 57 & $G B A$ & c.1309delG \\
\hline & 13 & Female & 41 & $G B A$ & p.Asp386Lys \\
\hline & 14 & Female & 49 & $G B A$ & $\mathrm{IVS} 2+1 \mathrm{~g}>\mathrm{A}$ \\
\hline Mean \pm SD & & & $48.5 \pm 6.7$ & & \\
\hline \multirow[t]{5}{*}{ Controls } & 1 & Male & 53 & None & - \\
\hline & 2 & Female & 45 & None & - \\
\hline & 3 & Female & 59 & None & - \\
\hline & 4 & Female & 46 & None & - \\
\hline & 5 & Female & 45 & None & - \\
\hline Mean \pm SD & & & $49.6 \pm 6.2$ & & \\
\hline
\end{tabular}

SD: standard deviation.

numbers were no greater than 10 .

\section{Confirmation of the genotypes in fibroblasts}

DNA was extracted from fibroblasts using a standard phenolchloroform method. All point mutations were confirmed by using both direct sequencing of the interested exons, and appropriated PCR-RFLPs methods (Table 1). Direct sequencing of all exons and exon-intron boundaries of GBA and PARK2 genes was performed in all DNA samples of the control subjects in order to exclude the presence of any point variants in the studied genes. PARK2 exon rearrangement was assessed in all DNA samples by using both semi-quantitative fluorescently labeled multiplex PCR and denaturing high-performance liquid chromatography (DHPLC) as prior protocols described $[20,21]$.

\section{Assessment of mitochondrial respiratory chain function}

Activities of respiratory chain enzymes (complex I, NADH dehydrogenase; complex II, succinate-coenzyme Q reductase; complex III, cytochrome c reductase; complex IV, cytochrome c oxidase; and complex V, ATP synthase) and citrate synthase were determined using enzyme activity microplate assay kits (catalog no. ab109721, ab109908, ab124537, ab109909, ab109714, and ab119692; Abcam, Cambridge, MA, USA) in whole cell lysates at an immunocapture based manner, according to the manufacturer's instructions. The amount of protein was determined using a BCA protein assay. The enzyme activity was expressed as the change in absorbance per minute (mOD/min) per amount of sample loaded into the microplate well. The sample rate was compared with the rate of the control sample and with the rate of the background to get the relative enzyme activity. The enzyme activity was determined by reading the optical density using a spectrophotometer (Molecular Devices, Sunnyvale, CA, USA). The rate was determined by calculating the slope between two points within the linear region. To correct for mitochondrial volume, complex I - V activities were normalized with the activity of citrate synthase, an exclusive marker of the mitochondrial matrix. All measurements were performed in triplicate and in three independent runs per sample.

\section{Statistical analysis}

Results of enzymatic activities were expressed as mean \pm standard error of measurement (SEM) and scatter plots. Statistical analysis was done by the GraphPad Prism 5 Software for Windows (GraphPad Software, Inc., San Diego, CA, USA). 
Table 3. Enzymatic Activities (Mean \pm SEM) of Complexes I - V Corrected by Citrate Synthase Activity

\begin{tabular}{lllllll}
\hline & Control $(\mathbf{N}=\mathbf{5})$ & \multicolumn{5}{c}{ Patients } \\
\cline { 3 - 7 } & & $\begin{array}{l}\text { All patients } \\
(\mathbf{N = 1 4 )}\end{array}$ & $\begin{array}{l}\text { No mutation } \\
(\mathbf{N = 7})\end{array}$ & $\begin{array}{l}\text { All mutations } \\
(\mathbf{N}=7)\end{array}$ & $\begin{array}{l}\text { PARK2 } \\
(\mathbf{N}=\mathbf{3})\end{array}$ & $\begin{array}{l}G B A \\
(\mathbf{N}=\mathbf{4})\end{array}$ \\
\hline Complex I $\left(/ \times 10^{3}\right)$ & $8.92 \pm 0.55$ & $10.78 \pm 0.45$ & $11.48 \pm 0.50$ & $10.09 \pm 0.68$ & $9.44 \pm 0.71$ & $10.57 \pm 1.10$ \\
Complex II $\left(/ \times 10^{3}\right)$ & $107.9 \pm 11.57$ & $117.4 \pm 7.45$ & $122.1 \pm 13.77$ & $112.6 \pm 6.40$ & $125.70 \pm 6.25$ & $102.70 \pm 6.90$ \\
Complex III $\left(/ \times 10^{2}\right)$ & $53.11 \pm 3.69$ & $51.69 \pm 3.00$ & $48.88 \pm 2.60$ & $54.50 \pm 5.43$ & $47.42 \pm 11.66$ & $59.81 \pm 3.64$ \\
Complex IV $\left(/ \times 10^{3}\right)$ & $27.15 \pm 2.28$ & $29.30 \pm 1.31$ & $31.61 \pm 2.04$ & $26.99 \pm 1.21$ & $27.61 \pm 1.35$ & $26.52 \pm 2.01$ \\
Complex V $(/ \times 10)$ & $34.13 \pm 2.39$ & $32.95 \pm 2.06$ & $35.08 \pm 3.96$ & $30.83 \pm 1.08$ & $31.63 \pm 2.41$ & $30.23 \pm 0.94$ \\
Citrate synthase $(\mathrm{mOD} / \mathrm{min})$ & $13.32 \pm 0.19$ & $12.66 \pm 0.13$ & $12.46 \pm 0.14$ & $12.87 \pm 0.21$ & $12.97 \pm 0.30$ & $12.79 \pm 0.31$ \\
\hline
\end{tabular}

SEM: standard error of measurement.

The Student's two-sample $t$-test was used for comparisons between patients and controls, or between mutant and nonmutant groups. A P less than 0.05 was considered statistically significant.

\section{Results}

Phenotypic and genotypic data of PD patients and controls are summarized in Table 2. Patients (mean age $\pm \mathrm{SD}, 48.5 \pm 6.7$ years) included seven without PARK2 and GBA mutations, three with PARK2 mutation, and four with GBA mutation. Five age-matched controls had a mean age $\pm \mathrm{SD}$ of $49.6 \pm 6.2$ years.

Enzymatic activities of mitochondrial respiratory chain, complex I - V, for patients and controls are shown in Table 3. All of the enzymatic activities were corrected with citrate synthase activity. Scatter plots of the level of enzymatic activities are shown in Figure 1. As shown, complex V activity seemed to be lower in PD patients than controls. Among patients, activities of complexes I, II, IV and V seemed to be lower in the mutant group than those without mutation. Among patients with mutations, the mutant GBA group showed slightly lower activities of complexes II, IV, and V than the mutant PARK2 group.

\section{Discussion}

In this study, we investigate whether enzymatic activities of mitochondrial respiratory chain complexes I - V are affected in skin fibroblast cultures of Thai PD patients with GBA and heterozygous PARK2 mutations. The effect of single heterozygous mutations in PARK2 and GBA is still unclear. Although we for the first time report enzymatic activities of mitochondrial respiratory chain complexes I - V in skin fibroblasts of GBA-mutant patients, the slightly decreased activities of complexes II, IV, and especially V, compared with controls, need to be confirmed in a larger scale of patients. Complex $\mathrm{V}$ is involved in the ATP synthesis, and is very crucial in the energetic metabolism of the cells. Impairment of mitochondrial functions caused by GBA inhibition has been demonstrated in the human dopaminergic cell line [16], and the finding of mitochondrial dysfunction in skin fibroblasts of GBA-mutant patients, if confirmed, would facilitate research on the mechanism of GBA mutation-related PD.

Our findings on activities of mitochondrial respiratory chain complexes in patients with PARK2 mutation $(\mathrm{N}=3)$ are similar to those of Grunewald et al $(\mathrm{N}=6)$ [13], showing no significant differences in complexes I, II + III, and IV activities, compared with controls. However, Grunewald et al measured ATP synthesis rates and intracellular ATP concentrations, and revealed a significant reduction of both parameters compared to controls, suggesting an existence of abnormal mitochondrial function in skin fibroblasts of PD patients with PARK2 mutation.

Del Hoyo et al reported insignificant differences of complexes I - IV activities between PD patients $(\mathrm{N}=20)$ and control groups, in skin fibroblast cultures, which is similar to our results on non-mutant $\mathrm{PD}$ patients $(\mathrm{N}=7)$ [15]. However, a decreased activity of complex $\mathrm{V}$ was observed in del Hoyo et al's work. Compared PD patients with and without GBA or PARK2 mutation, our results showed no significant differences of complexes I - V activities, although a slightly decreased activity was observed for complexes IV and $\mathrm{V}$ in the mutation group.

The discrepancies of the results of complexes I - V activities among skin fibroblast studies in PD patients may be related to the difference in methods used in assessing the mitochondrial respiratory chain complexes, the presence or absence of PDrelated mutations, types of mutation, types of samples (whole cell extract or mitochondrial extract), and sample size. However, evidence that mutations of PD-related genes can cause functional and morphological abnormalities of mitochondria in skin fibroblasts has been reported, such as for PARK2, and PINK1 [13, 22, 23].

\section{Conclusion}

A mild decrease in some mitochondrial respiratory chain complexes, especially complex V, was detectable in skin fibroblasts of patients with GBA mutation. However, the measurement of steady-state total ATP present in fibroblasts is required to support this finding. Another point of note, although with a negative result, is related to the fact that this is the first report 

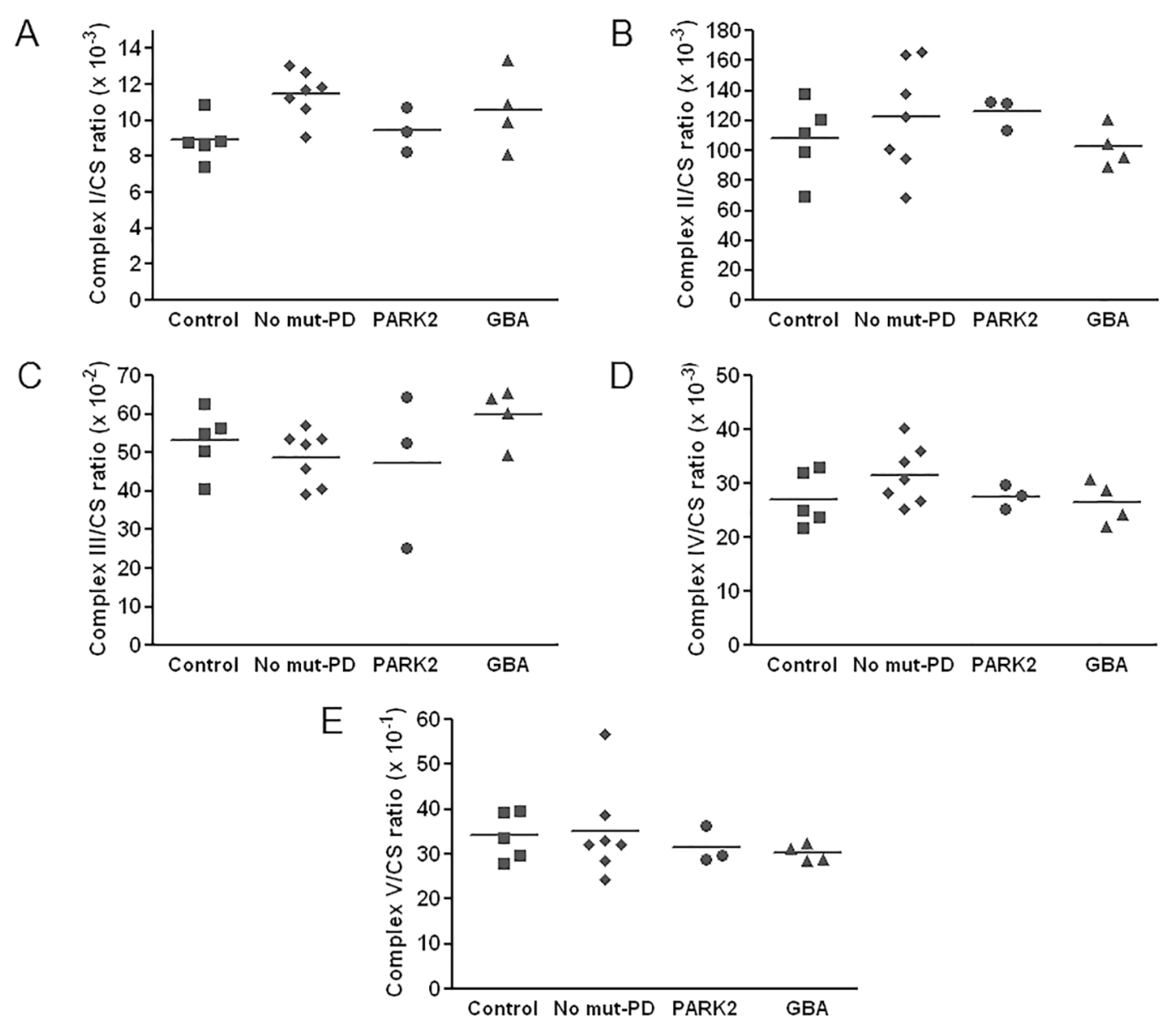

Figure 1. Scatter plots of values of enzymatic activities of complexes I - V in Parkinson's disease (PD) patients and in controls. All respiratory chain enzyme activities are normalized to the activity of citrate synthase (CS). Horizontal bars represent the mean values.

on respiratory chain enzymatic activities in skin fibroblasts of GBA-mutant individuals. Further validation in a larger patient population is required, using a variety of analyzes for mitochondrial functions. Target specificity of mutant GBA on respiratory complexes also remains to be investigated. The present results suggest that a skin fibroblast culture may be a useful tool to investigate the mechanisms underlying how GBA mutation leads to impairment of the energetic metabolism of the cells of peripheral tissues in patients with PD.

\section{Disclosure}

This research is under the research framework of Mahidol University.

\section{Financial Disclosures}

This research was supported by grants from Faculty of Science, Faculty of Medicine, Ramathibodi Hospital, Mahidol University (to P. Dharmasaroja and T. Pulkes), Thailand Research Fund
Royal Golden Jubilee Ph.D. Scholarship (PHD/0058/2556 to K. Khwanraj), and partly, Thailand Research Fund and Mahidol University (IRG5780011 to P. Dharmasaroja).

\section{Conflicts of Interest}

All authors declare no conflict of interest.

\section{References}

1. Lucking CB, Durr A, Bonifati V, Vaughan J, De Michele G, Gasser T, Harhangi BS, et al. Association between early-onset Parkinson's disease and mutations in the parkin gene. N Engl J Med. 2000;342(21):1560-1567.

2. Nakaso K, Adachi Y, Yasui K, Sakuma K, Nakashima $\mathrm{K}$. Detection of compound heterozygous deletions in the parkin gene of fibroblasts in patients with autosomal recessive hereditary parkinsonism (PARK2). Neurosci Lett. 2006;400(1-2):44-47.

3. Auburger G, Klinkenberg M, Drost J, Marcus K, Mo- 
rales-Gordo B, Kunz WS, Brandt U, et al. Primary skin fibroblasts as a model of Parkinson's disease. Mol Neurobiol. 2012;46(1):20-27.

4. Sato C, Morgan A, Lang AE, Salehi-Rad S, Kawarai T, Meng Y, Ray PN, et al. Analysis of the glucocerebrosidase gene in Parkinson's disease. Mov Disord. 2005;20(3):367370.

5. Aharon-Peretz J, Rosenbaum H, Gershoni-Baruch R. Mutations in the glucocerebrosidase gene and Parkinson's disease in Ashkenazi Jews. N Engl J Med. 2004;351(19):1972-1977.

6. Spitz M, Rozenberg R, Pereira Lda V, Reis Barbosa E. Association between Parkinson's disease and glucocerebrosidase mutations in Brazil. Parkinsonism Relat Disord. 2008;14(1):58-62.

7. De Marco EV, Annesi G, Tarantino P, Rocca FE, Provenzano G, Civitelli D, Ciro Candiano IC, et al. Glucocerebrosidase gene mutations are associated with Parkinson's disease in southern Italy. Mov Disord. 2008;23(3):460463.

8. Neumann J, Bras J, Deas E, O'Sullivan SS, Parkkinen L, Lachmann RH, Li A, et al. Glucocerebrosidase mutations in clinical and pathologically proven Parkinson's disease. Brain. 2009;132(Pt 7):1783-1794.

9. Sidransky E, Nalls MA, Aasly JO, Aharon-Peretz J, Annesi G, Barbosa ER, Bar-Shira A, et al. Multicenter analysis of glucocerebrosidase mutations in Parkinson's disease. N Engl J Med. 2009;361(17):1651-1661.

10. Brockmann K, Berg D. The significance of GBA for Parkinson's disease. J Inherit Metab Dis. 2014;37(4):643648.

11. Schapira AH. Glucocerebrosidase and Parkinson disease: Recent advances. Mol Cell Neurosci. 2015;66(Pt A):3742.

12. McNeill A, Magalhaes J, Shen C, Chau KY, Hughes D, Mehta A, Foltynie T, et al. Ambroxol improves lysosomal biochemistry in glucocerebrosidase mutation-linked Parkinson disease cells. Brain. 2014;137(Pt 5):1481-1495.

13. Grunewald A, Voges L, Rakovic A, Kasten M, Vandebona H, Hemmelmann C, Lohmann K, et al. Mutant Parkin impairs mitochondrial function and morphology in human fibroblasts. PLoS One. 2010;5(9):e12962.

14. Mortiboys H, Thomas KJ, Koopman WJ, Klaffke S,
Abou-Sleiman P, Olpin S, Wood NW, et al. Mitochondrial function and morphology are impaired in parkin-mutant fibroblasts. Ann Neurol. 2008;64(5):555-565.

15. del Hoyo P, Garcia-Redondo A, de Bustos F, Molina JA, Sayed Y, Alonso-Navarro H, Caballero L, et al. Oxidative stress in skin fibroblasts cultures from patients with Parkinson's disease. BMC Neurol. 2010;10:95.

16. Cleeter MW, Chau KY, Gluck C, Mehta A, Hughes DA, Duchen M, Wood NW, et al. Glucocerebrosidase inhibition causes mitochondrial dysfunction and free radical damage. Neurochem Int. 2013;62(1):1-7.

17. Pulkes T, Choubtum L, Chitphuk S, Thakkinstian A, Pongpakdee S, Kulkantrakorn K, Hanchaiphiboolkul $\mathrm{S}$, et al. Glucocerebrosidase mutations in Thai patients with Parkinson's disease. Parkinsonism Relat Disord. 2014;20(9):986-991.

18. Pulkes T, Papsing C, Thakkinstian A, Pongpakdee S, Kulkantrakorn K, Hanchaiphiboolkul S, Tiamkao S, et al. Confirmation of the association between LRRK2 R1628P variant and susceptibility to Parkinson's disease in the Thai population. Parkinsonism Relat Disord. 2014;20(9):1018-1021.

19. Hughes AJ, Daniel SE, Kilford L, Lees AJ. Accuracy of clinical diagnosis of idiopathic Parkinson's disease: a clinico-pathological study of 100 cases. J Neurol Neurosurg Psychiatry. 1992;55(3):181-184.

20. Lucking CB, Brice A. Semiquantitative PCR for the detection of exon rearrangements in the Parkin gene. Methods Mol Biol. 2003;217:13-26.

21. Dehainault C, Lauge A, Caux-Moncoutier V, Pages-Berhouet S, Doz F, Desjardins L, Couturier J, et al. Multiplex PCR/liquid chromatography assay for detection of gene rearrangements: application to RB1 gene. Nucleic Acids Res. 2004;32(18):e139.

22. Klinkenberg M, Thurow N, Gispert S, Ricciardi F, Eich F, Prehn JH, Auburger G, et al. Enhanced vulnerability of PARK6 patient skin fibroblasts to apoptosis induced by proteasomal stress. Neuroscience. 2010;166(2):422-434.

23. Hoepken HH, Gispert S, Morales B, Wingerter O, Del Turco D, Mulsch A, Nussbaum RL, et al. Mitochondrial dysfunction, peroxidation damage and changes in glutathione metabolism in PARK6. Neurobiol Dis. 2007;25(2):401-411. 